\title{
Lipid Profiles in Wheat Cultivars Resistant and Susceptible to Tan Spot and the Effect of Disease on the Profiles
}

\author{
Dongwon Kim, Richard Jeannotte, Ruth Welti, and William W. Bockus
}

First and fourth authors: Department of Plant Pathology, Kansas State University, Manhattan 66506; second author: Department of Population Health and Reproduction, University of California, Davis 95616; and third author: Kansas Lipidomics Research Center, Division of Biology, Kansas State University, Manhattan 66506.

Accepted for publication 24 September 2012.

\begin{abstract}
Kim, D., Jeannotte, R., Welti, R., and Bockus, W. W. 2013. Lipid profiles in wheat cultivars resistant and susceptible to tan spot and the effect of disease on the profiles. Phytopathology 103:74-80.

Lipid profiles in wheat leaves and the effects of tan spot on the profiles were quantified by mass spectrometry. Inoculation with Pyrenophora tritici-repentis significantly reduced the amount of leaf lipids, including the major plastidic lipids monogalactosyldiacylglycerol (MGDG) and digalactosyldiacylglycerol (DGDG), which together accounted for $89 \%$ of the mass spectral signal of detected lipids in wheat leaves. Levels of these lipids in susceptible cultivars dropped much more quickly during

infection than those in resistant cultivars. Furthermore, cultivars resistant or susceptible to tan spot displayed different lipid profiles; leaves of resistant cultivars had more MGDG and DGDG than susceptible ones, even in noninoculated plants. Lipid compositional data from leaves of 20 noninoculated winter wheat cultivars were regressed against an index of disease susceptibility and fitted with a linear model. This analysis demonstrated a significant relationship between resistance and levels of plastidic galactolipids and indicated that cultivars with high resistance to tan spot uniformly had more MGDG and DGDG than cultivars with high susceptibility. These findings suggest that lipid composition of wheat leaves may be a determining factor in the resistance response of cultivars to tan spot.
\end{abstract}

Wheat is an important source of calories for humans and animals. In the United States, wheat is consumed by humans in various products such as bread, pasta, and pizza. Worldwide wheat production in 2009 was estimated at 25 billion bushels, and 2.2 billion bushels were produced in the United States. Kansas produced 360 million bushels of wheat in 2010 (34) and over 400 million bushels in 12 of the last 33 years. However, exposure of wheat to abiotic stresses, such as drought, heat, and cold, can affect wheat production. Biotic agents, including insects, bacteria, and fungi, also reduce yields. Exposure to abiotic and biotic stresses can affect both wheat quality and quantity.

Tan spot, which is caused by the fungus Pyrenophora triticirepentis (Died.) Drechsler, is one of the major foliar diseases of wheat worldwide (7), causing crop losses of up to $50 \%(27,29)$. Increases in disease incidence have been attributed to changes in cultural practices (16) such as shifts from conventional tillage to conservation and zero tillage, shorter crop rotations, continuous wheat cultivation, and the use of highly susceptible cultivars (4). The fungus overwinters as fruiting bodies called pseudothecia that develop on the previous season's infected wheat residue on and above the soil surface. Pseudothecia release sexual spores (ascospores) in the spring, inducing the first infections of the growing season. Asexual spores (conidia) are produced on crop residue and from leaf spots. Conidia are dispersed by wind and germinate to infect wheat in a wide range of temperatures but infection requires continual leaf wetness for at least $6 \mathrm{~h}$ (22). During the growing season, many conidia can form in the lesions, serving as secondary inoculum to produce an epidemic (21).

Tan spot produces two main phenotypic symptoms on wheat leaves: necrosis and chlorosis. These symptoms are induced by at least three host-specific toxins designated Ptr ToxA, Ptr ToxB,

Corresponding author: W. W. Bockus; E-mail address: Bockus@ksu.edu

http://dx.doi.org/10.1094/PHYTO-05-12-0099-R

(c) 2013 The American Phytopathological Society and Ptr ToxC (30). These toxins are important in the tan spotwheat pathosystem. The eight races of the fungus are classified based on virulence patterns, related to the putative production or nonproduction of these toxins, as deduced by effect of the fungi on a set of differential wheat lines or cultivars (17). Ptr ToxA is the best-characterized toxin and was the first to be isolated $(1,32,33)$. It is responsible for the necrotic symptom on sensitive wheat genotypes and is a $13.2-\mathrm{kDa}$ protein encoded by the ToxA gene $(2,4)$. Ptr ToxB is also a small $(6.6-\mathrm{kDa})$ protein molecule and is encoded by the ToxB gene; it induces chlorosis on sensitive wheat genotypes (31). Ptr ToxC also induces chlorosis but on different wheat lines or cultivars (10). It is not proteinaceous like Ptr ToxA and Ptr ToxB but is a nonionic, polar, low-molecularmass molecule (9).

In successful infections, the fungal ascospores or conidia germinate by forming a germ tube under free moisture when they land on wheat leaves. The germ tube produces a penetration peg which facilitates penetration of the epidermal cell. Infection can be either direct or indirect, such as through stomata, with the penetration peg forming a vesicle inside the leaf. Intercellular fungal hyphae grow and expand among the epidermal and mesophyll cells. The toxins produced by $P$. tritici-repentis enter cells and induce damage of cellular organelles (18) (http://www. apsnet.org/edcenter/intropp/lessons/fungi/ascomycetes/Pages/Tan Spot.aspx). Manning and Ciuffetti (20) suggest that Ptr ToxA is internalized in only sensitive wheat cultivars and, once internalized, it localizes to the chloroplasts. The Ptr ToxA protein is able to cross the plant plasma membrane from the apoplastic space to the interior of a plant cell, leading to cell death. Ciuffetti el al. (3) report that Ptr ToxA leads to a light-dependent reactive oxygen species accumulation that correlates with the presence of necrosis and modifies photosystem I and photosystem II in the absence of light. In the development of chlorosis in response to Ptr ToxB, the chlorophyll molecule is photooxidized and illuminated thylakoid membranes become unable to dissipate excess excitation energy (31). Kim et al. (15) report that Ptr ToxB inhibits photosynthesis 
in toxin-sensitive wheat lines and suggest that it induces alterations of the proteome level in host metabolism.

Lipids are often defined as biological compounds that are soluble in organic solvents (35). In practice, the term "lipids" also includes fatty-acid-containing compounds such as highly glycosylated glycolipids that have limited solubility in organic solvents, and excludes hydrophobic molecules such as hydrophobic peptides which can be otherwise classified. Lipids are important to plants for energy storage and as protective "waxy" surfaces on cells and plant leaves, stems, and roots (11). They form cell membranes, including those in which photosynthesis takes place, and serve as signal transduction messengers that influence plant growth, development, and response to stress (28). Polar glycerolipids are an important group of membrane-forming lipids that typically contain two long-chain fatty acids esterified to glycerol, with a polar headgroup also attached to the glycerol.

Plants are constantly exposed to both abiotic and biotic stresses. Plant membrane lipids, particularly polar glycerolipids, may change as plants respond to these stresses, and the lipid changes may be intimately involved in mediating plant stress responses. For example, levels of unsaturated fatty acyl chains, including linolenic acid, change in response to temperature, affecting membrane fluidity and the ability of a plant to tolerate extreme temperatures $(13,37)$. Linolenic acid also is involved in protein modifications in plants under heat stress (40). Freezing tolerance also is enhanced by the presence of an activity, encoded by the gene SENSITIVE TO FREEZING2 (SFR2), which remodels the outer chloroplast membrane (23). The enzyme transfers a galactosyl residue from a monogalactosyldiacylglycerol (MGDG) to a galactolipid accepter to form an oligogalactolipid and diacylglycerol. The biochemical activity of SFR 2 causes a compositional change in the membrane that is likely to increase bilayer physical stability during freezing (23). PA, formed from the hydrolysis of other phospholipids by the action of phospholipase D in plant stresses such as freezing and drought, can regulate the function of a number of proteins; for example, ABI1, a protein phosphatase involved in abscisic acid signaling $(36,41)$. Finally, membrane lipids undergo modifications that produce lipid-derived signal molecules, such as jasmonic acid, that are critical to plant wound and disease responses (28).

As described above, the toxins produced by $P$. tritici-repentis interact closely with host tissues. The toxins can cause necrotic cell death, inhibition of photosynthesis, and induction of chlorosis in wheat leaves. The interactions of the fungus and its toxins with plant leaves involve interactions with membranes. Thus, it is likely that there are interactions among the fungus, wheat lipids, and wheat proteins that regulate lipid composition. A working hypothesis for this research was that the fungus-host interaction affects wheat leaf lipid metabolism, resulting in different membrane lipid compositions between leaves of diseased and healthy wheat cultivars. Therefore, one goal of the research was to determine whether infection by $P$. tritici-repentis affects the lipid profiles of wheat leaves. A second goal was to determine whether there was a difference in leaf lipid profiles among wheat cultivars susceptible or resistant to tan spot.

\section{MATERIALS AND METHODS}

Experiment number 1 plant material. Wheat (Triticum aestivum L.) seed were planted and the resulting seedlings grown for 1 month in the greenhouse [ 20 to $28^{\circ} \mathrm{C}$, with supplemental light from high-pressure sodium lights $(400 \mathrm{~W})]$. Experiments were conducted between November and April. Seedlings were grown in racks holding 2.5-by-13-cm plastic tubes (Stuewe and Sons, Tangent, OR) filled with a mixture of steam-sterilized soil and vermiculite (50:50). There were 48 treatments arranged in a randomized complete block design with five replications. An experimental unit was a single plant growing in a tube. Treatments included three resistant and three susceptible cultivars, with or without inoculation with $P$. tritici-repentis, and four harvest times after inoculation. Resistant cultivars were 'Betty', 'Jagger', and 'Karl 92' and susceptible cultivars were 'Larned', 'Newton', and 'TAM 105'. Tan spot susceptibility or resistance ratings are shown in Table 1. Harvest times were 2, 4, 6, or 8 days after inoculation. The experiment was conducted twice.

Inoculum production and inoculation. Race 1 of $P$. triticirepentis was used. This race is the most abundant in the Great Plains of the United States, including Kansas, and produces ToxA and ToxC. Spores were produced by placing $0.5-\mathrm{cm}^{2}$ mycelial plugs of $P$. tritici-repentis from one-fourth-strength potato dextrose agar (1/4 PDA) in the center of plates of V-8 agar $(150 \mathrm{ml}$ of $\mathrm{V}-8$ juice, $3 \mathrm{~g}$ of $\mathrm{CaCO}_{3}, 15 \mathrm{~g}$ of agar, and $850 \mathrm{ml}$ of distilled water). Plates were incubated in the dark at 21 to $24^{\circ} \mathrm{C}$ for 5 days until the colony reached $\approx 5 \mathrm{~cm}$ in diameter. Aerial mycelia were knocked down with a sterile, bent-glass rod and plates were incubated in the light $\left(\approx 30 \mu \mathrm{E} \mathrm{s}^{-1} \mathrm{~m}^{-2}\right)$ at 21 to $24^{\circ} \mathrm{C}$ for $12 \mathrm{~h}$ to produce conidiophores and then in the dark at $16^{\circ} \mathrm{C}$ for $12 \mathrm{~h}$ to produce conidia. Plants were inoculated when 1 month old. Spore suspensions were quantified using a hemacytometer and adjusted with distilled water to 10,000 spores $/ \mathrm{ml}$. A spore suspension $(35 \mathrm{ml})$ was uniformly applied using a DeVilbis atomizer (Micromedics Inc., St. Paul, MN) per 30-by-60-cm rack holding two replications (96 tubes). Leaves were allowed to dry to stick spores to the leaves and the plants then placed into a mist chamber to maintain continual leaf wetness for $48 \mathrm{~h}$ at 20 to $28^{\circ} \mathrm{C}$. After the mist treatment, plants were returned to the greenhouse bench (20 to $28^{\circ} \mathrm{C}$ ).

Disease rating, harvest, and processing. At each sample time, leaves were rated for percent leaf area displaying chlorosis or necrosis (25). After rating for leaf area affected by disease, lipid extraction was carried out according to the protocols published by the Kansas Lipidomics Research Center $(5,39)$, with slight modifications as stated below. The first and second leaves of each plant were removed, quickly cut with scissors into $1-\mathrm{cm}$ pieces, and immersed in $6 \mathrm{ml}$ of preheated $\left(75^{\circ} \mathrm{C}\right)$ isopropanol with $0.01 \%$ butylated hydroxytoluene (BHT). The extraction solvent was in a 50-ml glass tube with a Teflon-lined screw cap. Leaf pieces were incubated in the $75^{\circ} \mathrm{C}$ isopropanol for at least $30 \mathrm{~min}$. In all, $3 \mathrm{ml}$ of chloroform and $1 \mathrm{ml}$ of water were then added to each tube and the tube was vortexed. Tubes were agitated in a shaking incubator at room temperature for $1 \mathrm{~h}$. The lipid extracts were transferred to

TABLE 1. Reaction of 20 selected winter wheat cultivars to tan spot

\begin{tabular}{llc}
\hline Entry number & Cultivar & Tan spot rating \\
\hline 1 & Red Chief & 2.60 \\
2 & Heyne & 2.80 \\
3 & Betty & 3.03 \\
4 & Karl 92 & 3.27 \\
5 & Jagger & 3.31 \\
6 & 2137 & 3.89 \\
7 & Victory & 4.10 \\
8 & Overley & 4.64 \\
9 & Wesley & 5.24 \\
10 & Protection CL & 6.12 \\
11 & Onaga & 6.45 \\
12 & Jagalene & 6.57 \\
13 & Abilene & 6.96 \\
14 & Ike & 8.02 \\
15 & 2180 & 8.40 \\
16 & Newton & 8.48 \\
17 & Larned & 8.68 \\
18 & TAM 105 & 8.87 \\
19 & Arkan & 9.01 \\
20 & Stanton & 9.05 \\
\hline
\end{tabular}

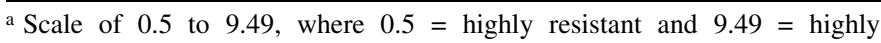
susceptible. Values are the means of at least five replicated phenotyping experiments (6; W. W. Bockus, unpublished data). 
another glass tube using a glass pipette. Four more extractions of lipid using $4 \mathrm{ml}$ of chloroform/methanol (2:1) with $0.01 \%$ BHT were carried out with shaking for $5 \mathrm{~h}$ or overnight until the leaves of the sample became white. Every sample had five extractions, including the one with the isopropanol. Samples were backwashed by adding $1 \mathrm{ml}$ of $1 \mathrm{M} \mathrm{KCl}$ to the combined extract, vortexing, centrifuging (10 min at 1,000 rpm), and removing the upper phase. A second backwash involved adding $2 \mathrm{ml}$ of water and repeating the backwash steps. All tubes were then evaporated under nitrogen. After complete evaporation, the extract was dissolved in $1 \mathrm{ml}$ of chloroform. All extracts were stored at $-75^{\circ} \mathrm{C}$ until lipid analysis. The remaining plant tissues were dried in an oven $\left(105^{\circ} \mathrm{C}\right)$ overnight and weighed (in milligrams) to determine the dry extracted tissue mass.

Quantification of lipids using mass spectrometry. An aliquot of the dissolved extract in $1 \mathrm{ml}$ of chloroform was used for lipid analysis by mass spectrometry. For analysis, 150 to $300 \mu \mathrm{l}$ of extract, dependent upon leaf dry weight, was combined with chloroform/methanol/300 mM ammonium acetate in water and internal standards. Complete information about internal standard additions, solvents, and mass spectral parameters are published (39, supplemental data at http://www.plantcell.org/content/suppl/ 2010/04/21/tpc.110.075333.DC1/Supplemental_Data_Final.pdf).

The lipid extracts were analyzed by a triple-quadrupole mass spectrometer (API 4000; Applied Biosystems, Foster City, CA). Injections to the mass spectrometer were at the rate of $30 \mu \mathrm{l} / \mathrm{min}$ using an autosampler with a 1-ml sample loop (LC Mini PAL; CTC Analytics AG, Zwingen, Switzerland).

Lipid profiles in resistant and susceptible cultivars (experiment number 2). Twenty winter wheat cultivars were selected based on Kansas State University extension ratings and data from unpublished phenotypic experiments and ranged from resistant to susceptible to tan spot (6) (Table 1). Seedlings were grown in the greenhouse as described above for 1 month. A single seed was sown in each tube. The design was a randomized complete block with 20 treatments (cultivars) and five replications (three plants per replication).

For lipid extraction, the five extractions were performed as described above. After these five chloroform/methanol-based extractions, $4 \mathrm{ml}$ of "solvent $\mathrm{H}$ " was added per tube and the tube

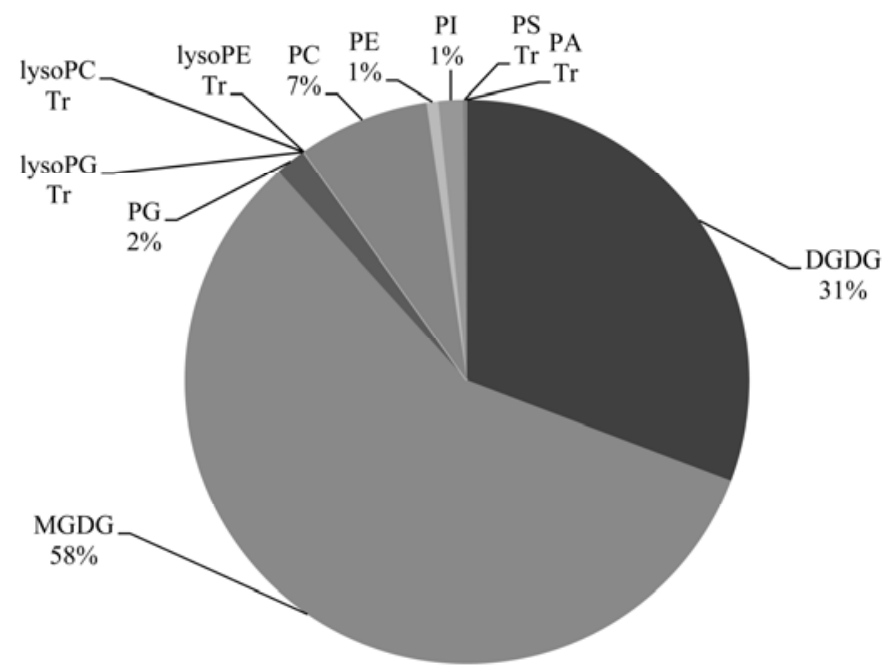

Fig. 1. Percentage of each lipid class (normalized mass spectral signal) detected in noninoculated wheat leaves; average of plants that were 30, 32, 34, and 36 days old. Lipids with "Tr" indicate detection at very low levels ("Trace"). Abbreviations: MGDG = monogalactosyldiacylglycerol, DGDG = digalactosyldiacylglycerol, $\mathrm{PC}=$ phosphatidylcholine, $\mathrm{PE}=$ phosphatidylethanolamine, $\mathrm{PG}=$ phosphatidylglycerol, $\mathrm{PI}=$ phosphatidylinositol, $\mathrm{PS}=$ phosphatidylserine, $\mathrm{PA}=$ phosphatidic acid, lysoPC = lysophosphatidylcholine, lysoPE = lysophosphatidylethanolamine, and lysoPG = lysophosphatidylglycerol. was incubated on a heating block at $60^{\circ} \mathrm{C}$ for $15 \mathrm{~min}$. Solvent $\mathrm{H}$ was prepared by mixing isopropanol/hexane/water (55:20:25, $\mathrm{vol} / \mathrm{vol} / \mathrm{vol}$ ) for $30 \mathrm{~min}$ with a magnetic stirrer. The mixture was allowed to settle for $30 \mathrm{~min}$, the upper phase was removed and discarded, the volume of the lower phase was measured, and BHT was added to a concentration of $0.01 \%$. The clear lower phase with BHT $0.01 \%$ is solvent $\mathrm{H}$. The solvent $\mathrm{H}$ extract of the wheat leaves was combined with previous chloroform/methanol extracts. The solvent $\mathrm{H}$ extraction, including the heating step, was repeated three more times, all extracts were combined, and no backwash was performed. Evaporation, drying, and weighing of leaves were as described above.

Data analysis. Data processing was carried out using a custom script and Applied Biosystems Analyst software. The amounts of lipid species were calculated using the software program Excel and the LipidomeDB Data Calculation Environment (http:// lipidome.bcf.ku.edu:9000/Lipidomics). Values are presented as mass spectral signal (intensity), normalized to internal standards, per milligram dry weight measured after lipid extraction, where a value of 1 refers to the same amount of mass spectral signal as $1 \mathrm{nmol}$ of internal standards. There were no significant experimental repetition-treatment interactions that would preclude combining the two repetitions of experiment number 1; therefore, the two repetitions were merged. Also, within a resistance class ("resistant" or "susceptible"), there were no significant cultivarinoculation interactions that would preclude combining data from the three resistant cultivars and from the three susceptible cultivars. Therefore, data within a resistance class were pooled for experiment number 1 . To determine the effect of resistance, inoculation, and time, linear models were fit to data using SAS (SAS Institute, Cary, NC). For each of the galactolipids (MGDG and digalactosyldiacylglycerol [DGDG]), four lines were compared. The four lines included resistant cultivars without inoculation, resistant cultivars with inoculation, susceptible cultivars without inoculation, and susceptible cultivars with inoculation. The amount of lipid was the independent variable and harvest time was the dependent variable. The resultant slopes of the lines were statistically compared $(P=0.05)$ using SAS. When the slopes of two lines were not significantly different, the equal-slopes model was used to compare the estimates of the intercepts $(P=0.05)$. For analysis of data from the experiment with 20 cultivars (experiment number 2), linear regression was used to determine the relationship between the amount of lipid and cultivar rating for tan spot resistance or susceptibility.

\section{RESULTS}

Lipids detected in wheat leaves (experiment number 1). Two classes of galactolipids and nine classes of phospholipids in extracts from wheat leaves were detected by mass spectrometry (Fig. 1). The galactolipids were monogalactosyldiacylglycerol (MGDG) and digalactosyldiacylglycerol (DGDG), and the phospholipids were phosphatidylcholine (PC), phosphatidylethanolamine (PE), phosphatidylglycerol (PG), phosphatidylinositol (PI), phosphatidylserine (PS), phosphatidic acid (PA), lysophosphatidylcholine (LysoPC), lysophosphatidylethanolamine (LysoPE), and lysophosphatidylglycerol (LysoPG). Galactolipids were the major lipid components in wheat leaves, accounting for $89 \%$ of all lipid mass spectral signals, while the phospholipid classes were present in lesser amounts (Fig. 1). PC was the most abundant phospholipid class. In both galactolipid classes (MGDG and DGDG), the major molecular species was 36:6, which has two linolenic acid moieties (di18:3). Lipid analytical results in the repeated experiment were similar.

Lipid class composition for healthy versus diseased and resistant versus susceptible plants (experiment number 1). There were consistent differences in the amounts of galactolipids (MGDG and DGDG) between inoculated and noninoculated 
treatments. The susceptible cultivars showed a significantly faster decline of MGDG and DGDG with inoculation when compared with the noninoculated treatments for the same susceptible cultivars (Figs. 2 and 3; Table 2; $\mathrm{S}+$ versus $\mathrm{S}_{-}$). The negative slope of the linear model was 3.6 and 5.0 times steeper for the inoculated treatments versus the noninoculated treatments for MGDG and DGDG, respectively. Similar large differences in the rate of reduction of galactolipids were seen when comparing the slopes of the inoculated susceptible cultivars with those of the inoculated or noninoculated resistant cultivars (Figs. 2 and 3; Table 2; S+ versus $\mathrm{R}+$ or $\mathrm{R}-$ ).

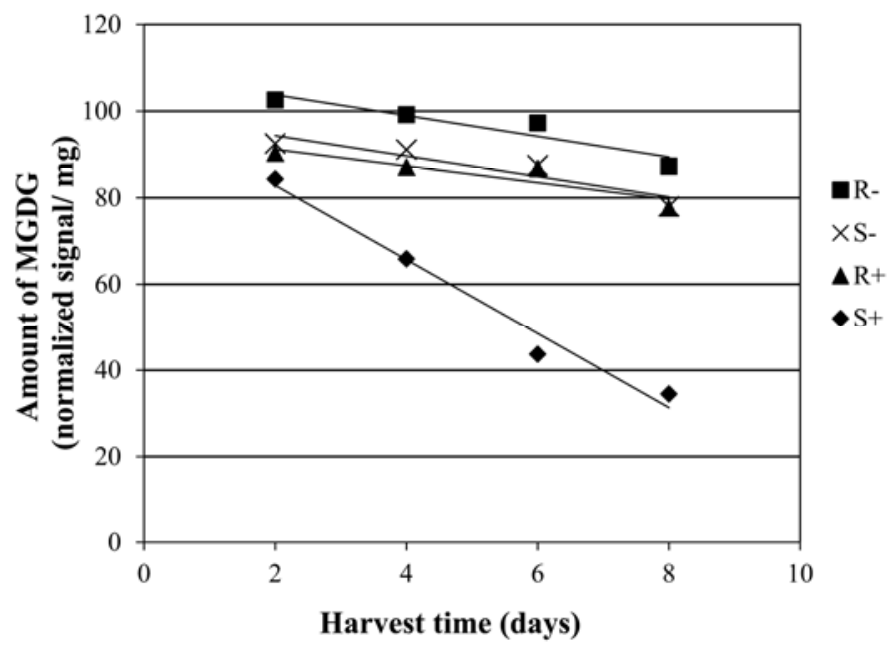

Fig. 2. Amount of monogalactosyldiacylglycerol (MGDG) in wheat leaves (normalized mass spectral signal per dry leaf mass) over time. One-month-old plants were inoculated or left noninoculated at day 0 . Abbreviations: $\mathrm{R}-=$ average of three resistant wheat cultivars ('Betty', 'Jagger', and 'Karl 92') without inoculation, $\mathrm{S}_{-}=$average of three susceptible cultivars ('Larned', 'Newton', and 'TAM 105') without inoculation, $\mathrm{R}+=$ resistant cultivars with inoculation, and $\mathrm{S}+=$ susceptible cultivars with inoculation. Each data point is the mean of three cultivars each with five replications. Equations for the trend lines and $P$ values for significance of the slopes different from zero are as follows R-, $Y=-2.39 X+108.53(P=0.0963)$; S,$- Y=-2.37 X+99.12(P=$ $0.1054) ; \mathrm{R}+, Y=-1.91 X+94.95(P=0.1847) ;$ and $\mathrm{S}+, Y=-8.61 X+100.10$ $(P<0.0001)$.
For MGDG, the slopes of the lines for the noninoculated susceptible cultivars, the inoculated resistant cultivars, and the noninoculated resistant cultivars were not significantly different from each other or from zero (Fig. 2; Table 2; $\mathrm{S}-, \mathrm{R}+, \mathrm{R}-$ ). However, the line for the noninoculated resistant cultivars was significantly above the line for the noninoculated susceptible cultivars (Table 2; $\mathrm{R}-$ versus $\mathrm{S}-$ ).

For DGDG, the slopes of the lines for the noninoculated susceptible cultivars, the inoculated resistant cultivars, and the noninoculated resistant cultivars were not significantly different from each other (Fig. 3; Table 2; - -, R+, R-). With this galactolipid,

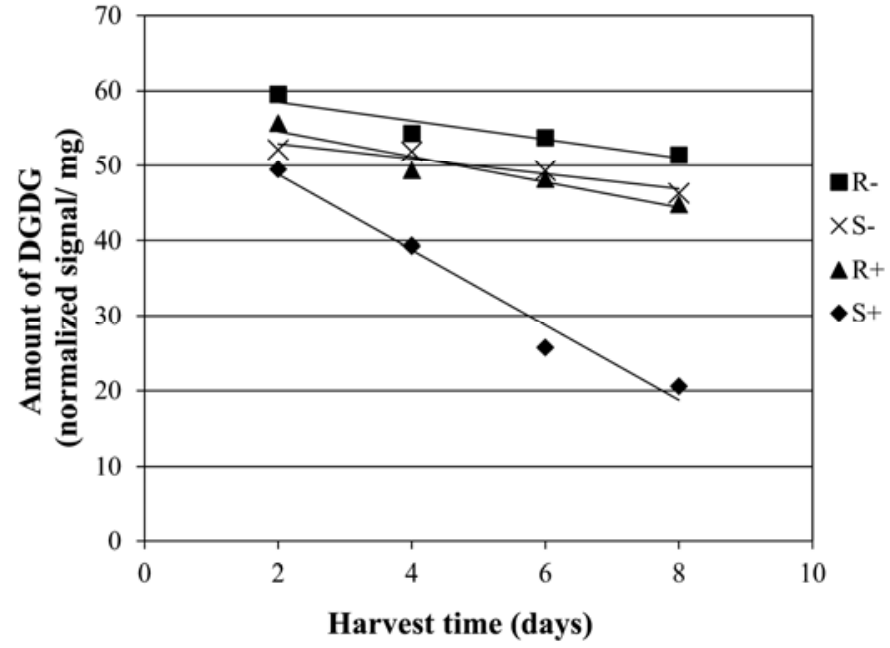

Fig. 3. Amount of digalactosyldiacylglycerol (DGDG) in wheat leaves (normalized mass spectral signal per dry leaf mass) over time. One-month-old plants were inoculated or left noninoculated at day 0 . Abbreviations: $\mathrm{R}-=$ average of three resistant wheat cultivars ('Betty', 'Jagger', and 'Karl 92') without inoculation, $\mathrm{S}_{-}=$average of three susceptible cultivars ('Larned', 'Newton', and 'TAM 105') without inoculation, $\mathrm{R}+=$ resistant cultivars with inoculation, and $\mathrm{S}+=$ susceptible cultivars with inoculation. Each data point is the mean of three cultivars each with five replications. Equations for the trend lines and $P$ values for significance of the slopes different from zero are as follows: R-, $Y=-1.25 X+61.02(P=0.0367) ; \mathrm{S}-, Y=-1.01 X+54.95(P=$ $0.0965) ; \mathrm{R}+, Y=-1.70 X+57.96(P=0.0047) ;$ and $\mathrm{S}+, Y=-5.02 X+58.85$ $(P<0.0001)$

TABLE 2. Statistical $P$ values for the comparison of slopes (in parentheses) and estimates of the intercepts (in parentheses) for the amount of monogalactosyldiacylglycerol (MGDG) or digalactosyldiacylglycerol (DGDG) regressed against harvest time for wheat cultivars resistant and susceptible to tan spot (lines shown in Figures 2 and 3$)^{\mathrm{a}}$

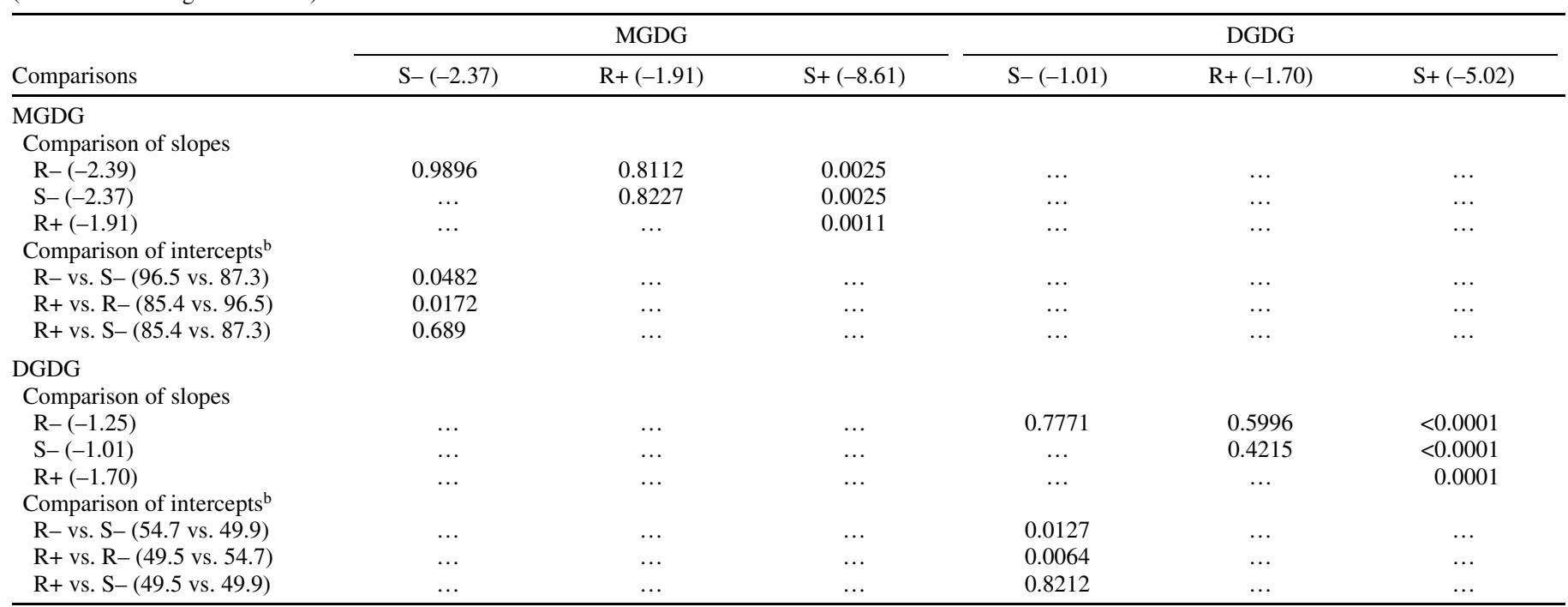

${ }^{\mathrm{a}} \mathrm{R}-=$ wheat cultivars resistant to tan spot without inoculation, $\mathrm{S}-=$ susceptible cultivars without inoculation, $\mathrm{R}+=$ resistant cultivars with inoculation, and $\mathrm{S}+=$ susceptible cultivars with inoculation. Slopes (normalized signal/milligram/day) and estimates of the intercepts are shown in parentheses.

${ }^{\mathrm{b}}$ Estimates of the intercepts were only compared for those pairings where the slopes of the lines were not significantly different. 
the slopes for the inoculated resistant cultivars and the noninoculated resistant cultivars were significantly different from zero but they were not very steep (Fig. 3; R+, R-). Although the slopes were not significantly different from each other, the line for the noninoculated resistant cultivars was significantly above the line for the noninoculated susceptible cultivars (Fig. 3; Table 2; Rversus $\mathrm{S}-$ ).

Correlation of lipid amount with tan spot resistance (experiment number 2). Results from experiment number 1 with six cultivars showed that noninoculated resistant cultivars had higher amounts of MGDG and DGDG compared with the noninoculated susceptible cultivars (Figs. 2 and 3; Table 2). Therefore, a second, expanded experiment involving 20 cultivars was conducted to corroborate that preliminary finding. The amounts of the major lipids were regressed against the disease phenotype rating for the cultivars. The cultivars had a range of reaction to tan spot from resistant to highly susceptible (Table 1 ). Figure 4 shows a significant $(P<0.0001)$ negative correlation between the amount of MGDG and the susceptibility rating. As the extension rating increased, the amount of MGDG decreased. There was a similar significant $(P<$ $0.0001)$ negative correlation between the amount of DGDG and tan spot extension rating; the higher the rating, the lower the amount of DGDG (Fig. 5).

\section{DISCUSSION}

The results presented here give evidence that a biotic stress can profoundly affect lipid profiles in plants. The results are the first documentation of the influence of the wheat leaf spot disease tan spot on lipids in wheat leaves. When comparing lipid profiles in diseased versus healthy plants, tan spot resulted in significant changes in lipid classes in some of the detected lipids (Table 2). Healthy wheat leaves had more of the lipids MGDG and DGDG. Furthermore, reductions of $>50 \%$ were observed for both lipids in inoculated susceptible cultivars 8 days after inoculation (Figs. 2 and $3 ; \mathrm{S}+$ ). It is unknown whether the fungus itself degraded the lipids or whether it induced plant enzymes to degrade the lipids. Further research is needed to elucidate the answer to that question.

Plants interact with the biotic and abiotic environments and have systems to protect themselves against stresses. When exposed to stresses, their survival often depends on how fast they recognize and respond to these stresses (19). The plasma membrane of a plant cell is often the first component where plants interact with

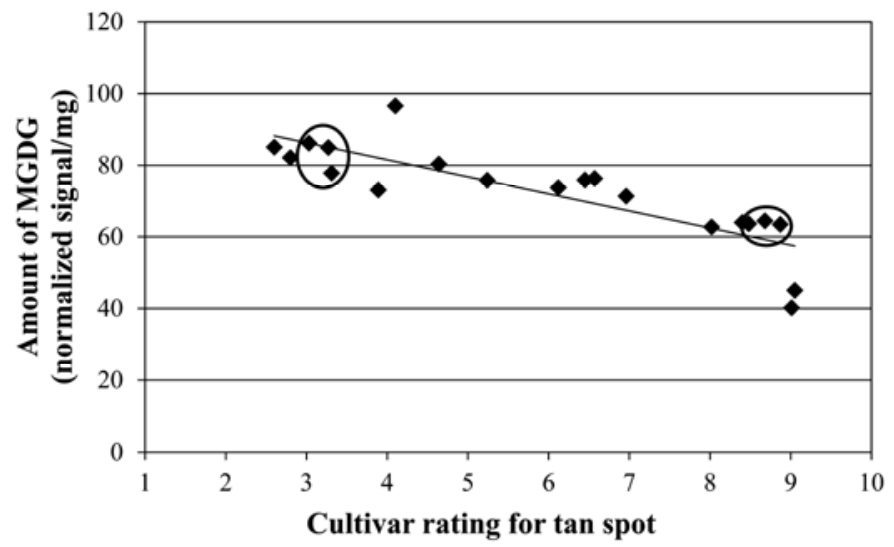

Fig. 4. Amount of monogalactosyldiacylglycerol (MGDG) versus the level of resistance to tan spot for noninoculated, 28-day-old seedling leaves of 20 winter wheat cultivars. Rating values (Table 1) are on a 0.5 to 9.49 scale, where $0.5=$ highly resistant to tan spot and $9.49=$ highly susceptible. Each data point is the mean of five replications for a single cultivar. Linear equation is: $Y=-4.77 X+100.63$ (adjusted $R^{2}=0.6815, N=20$, and $P<0.0001$ ). Three resistant and three susceptible cultivars that were used in experiment number 1 are circled. environmental stresses. Early events in the interaction between plants and environmental stresses can involve activities such as a kinase signal transduction pathway, phytohormones, and the production of reactive oxygen species in the plasma membrane (19). During these interactions, the composition of lipids in the cell membrane is changed.

There have been many studies involving changes in lipid profiles in plant cell membranes $(13,23,37,40)$. However, most previous studies have focused on changes in plant lipid composition due to abiotic stresses. Important findings in membrane biology concern the relationship between lipid composition and how plants adjust to temperature stress (38). In this regard, unsaturated fatty acids are linked to biochemical and physiological changes in plants exposed to chilling injury. Murata et al. (24) proposed a hypothesis that the level of unsaturated PG in chloroplast membranes determines the chilling sensitivity of plant species.

Results shown here are the first to correlate the amount of lipid moieties in wheat leaves with resistance level to tan spot. Wheat cultivars resistant or susceptible to tan spot showed different lipid profiles. For noninoculated treatments, the slopes of the lines for the galactolipids MGDG and DGDG were the same for resistant and susceptible cultivars and most were not significantly different from zero (Figs. 2 and 3; Table 2; R- versus $\mathrm{S}-$ ). This indicates that the levels did not change over time in healthy leaves. However, the estimates of the intercepts showed that there were higher $(P<0.05)$ levels of those lipids in the resistant cultivars (Table 2; 96.5 versus 87.3 for MGDG and 54.7 versus 49.9 for DGDG). Similarly, the rates of reduction due to tan spot for the galactolipids were significantly different between resistant and susceptible cultivars. Resistant cultivars had a significantly slower loss (larger slope) of MGDG and DGDG compared with susceptible cultivars (Figs. 2 and 3; Table 2; R+ versus S+). Therefore, these results suggest that lipids in susceptible wheat cultivars are influenced by tan spot more than those in resistant cultivars and the disease results in faster degradation of galactolipids in susceptible cultivars.

The experiment using 20 cultivars corroborated the above finding of higher levels of the major lipids in resistant cultivars in noninoculated plants. There was a significant $(P<0.0001)$ linear relationship between the amounts of MGDG and DGDG in noninoculated wheat leaves and the level of resistance to tan spot (Figs. 4 and 5). As the level of resistance increased (lower rating number), the level of MGDG and DGDG also increased. Using calculations from the linear equations, cultivars with a rating of 1

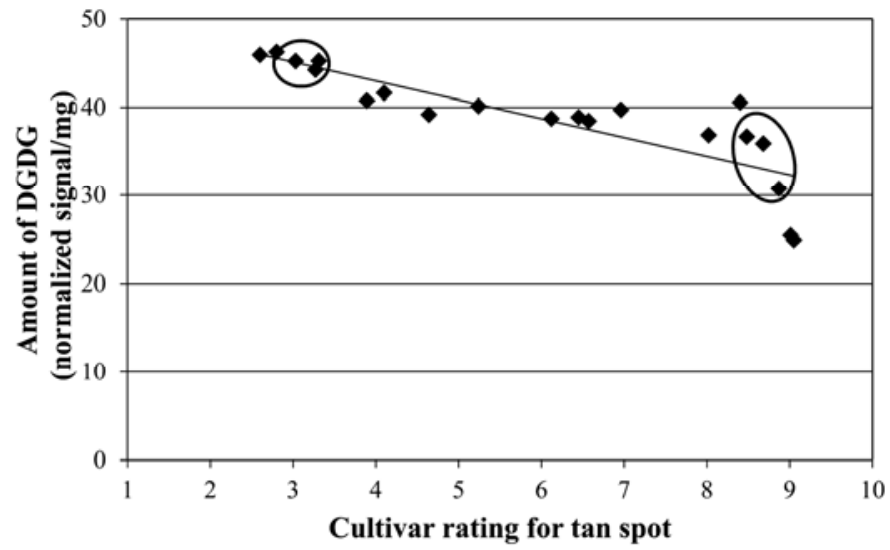

Fig. 5. Amount of digalactosyldiacylglycerol (DGDG) versus the level of resistance to tan spot for noninoculated, 28-day-old seedling leaves of 20 winter wheat cultivars. Rating values (Table 1 ) are on a 0.5 to 9.49 scale, where $0.5=$ highly resistant to tan spot and $9.49=$ highly susceptible. Each data point is the mean of five replications for a single cultivar. Linear equation is: $Y=$ $-2.13 X+51.52$ (adjusted $R^{2}=0.7041, N=20$, and $P<0.0001$ ). Three resistant and three susceptible cultivars that were used in experiment number 1 are circled. 
would have $66.1 \%$ more MGDG and 52.7\% more DGDG than cultivars with a rating of 9 (Figs. 4 and 5). MGDG and DGDG are major membrane constituents of chloroplasts and most abundant in plant leaves. They are indispensable for efficiency of photosynthetic light reactions. For example, Jarvis et al. (14) reported that MGDG synthase activity in the Arabidopsis mutant mgdl was reduced by $50 \%$ relative to that of the wild type, thus reducing the amount of MGDG and chlorophyll. Similarly, $d g d 1$ mutant plants contained $10 \%$ of the wild-type amount of DGDG and the mutant plants showed a strong reduction of photosynthetic capacity $(8,12,26)$.

Three kinds of host-specific toxins are involved in the pathogenesis of tan spot diseases. These toxins move into chloroplasts and interact with thylakoid membranes, causing inhibition of photosynthesis in wheat leaves $(15,20,31)$. Therefore, it may be that the toxins are responsible for the change in lipid profiles documented in this research. However, further research is needed to document this possibility.

In conclusion, data presented here are the first to quantify the effects of tan spot on lipids in wheat leaves. Data from timecourse experiments indicate that tan spot significantly reduced the amount of lipids, including the major lipids MGDG and DGDG, in leaves. This reduction was at a much higher rate for susceptible cultivars compared with resistant ones. Furthermore, data showed that cultivars resistant to tan spot have different lipid profiles when compared with susceptible cultivars. Resistant cultivars had more MGDG and DGDG than susceptible ones, even in noninoculated leaves. These findings are indirect evidence that the amounts of some lipids in wheat leaves are a determinant in the resistance response of cultivars to tan spot. However, further research is needed to corroborate this conclusion.

\section{ACKNOWLEDGMENTS}

This work was supported by the Kansas Wheat Commission and the Kansas Agricultural Experiment Station. Equipment acquisition at the Kansas Lipidomics Research Center was funded by the National Science Foundation (EPS 0236913 and DBI 0521587), Kansas Technology Enterprise Corporation, Kansas IDeA Networks of Biomedical Research Excellence (INBRE) of the National Institutes of Health (P20 RR16475), and Kansas State University. R. Jeannotte was supported by Kansas State University's Targeted Excellence program. Contribution number KAES number 12-320-J from the Kansas Agricultural Experiment Station. We thank M. R. Roth for technical assistance and S. Meier and T. C. Todd for statistical advice.

\section{LITERATURE CITED}

1. Ballance, G. M., Lamari, L., and Bernier, C. C. 1989. Purification and characterization of a host-selective necrosis toxin from Pyrenophora tritici-repentis. Physiol. Mol. Plant Pathol. 35:203-213.

2. Ballance, G. M., Lamari, L., Kowatsch, R., and Bernier, C. C. 1996. Cloning, expression and occurrence of the gene encoding the Ptr necrosis toxin from Pyrenophora tritici-repentis. Mol. Plant Pathol. http://www.bspp.org.uk/mppol/1996/1209ballance/

3. Ciuffetti, L. M., Manning, V. A., Pandelova, I., Betts, M. F., and Martinez, J. P. 2010. Host-selective toxins, Ptr ToxA and Ptr ToxB, as necrotrophic effectors in the Pyrenophora tritici-repentis-wheat interaction. New Phytol. 187:911-919.

4. Ciuffetti, L. M., Tuori, R. P., and Gaventa, J. M. 1997. A single gene encodes a selective toxin causal to the development of tan spot of wheat. Plant Cell 9:135-144.

5. Devaiah, S. P., Roth, M. R., Baughman, E., Li, M., Tamura, P., Jeannotte, R., Welti, R., and Wang, X. 2006. Quantitative profiling of polar glycerolipid species from organs of wild-type Arabidopsis and a phospholipase Dalpha1 knockout mutant. Phytochemistry 67:1907-1924.

6. De Wolf, E. D., Bockus, W. W., and Whitworth, J. R. 2011. Wheat variety disease and insect ratings 2011. Kans. Coop. Ext. Serv. Publ. MF-991.

7. De Wolf, E. D., Effertz, R. J., Al, S., and Francl, L. J. 1998. Vistas of tan spot research. Can. J. Plant Pathol. 20:349-370.

8. Dörmann, P., Hoffmann-Benning, S., Balbo, I., and Benning, C. 1995. Isolation and characterization of an Arabidopsis mutant deficient in the thylakoid lipid digalactosyl diacylglycerol. Plant Cell 7:1801-1810.
9. Effertz, R. J., Meinhardt, S. W., Anderson, J. A., Jordahl, J. G., and Francl, L. J. 2002. Identification of a chlorosis-inducing toxin from Pyrenophora tritici-repentis and the chromosomal location of an insensitivity locus in wheat. Phytopathology 92:527-533.

10. Gamba, F. M., Lamari, L., and Brule-Babel, A. L. 1998. Inheritance of race-specific necrotic and chlorotic reactions induced by Pyrenophora tritici-repentis in hexaploid wheats. Can. J. Plant Pathol. 20:401-407.

11. Graham, L. E., Graham, J. M., and Wilcox, L. W. 2003.Four types of primary compounds are the molecules of life. Pages 55-59 in: Plant Biology. Pearson Education, New Jersey.

12. Härtel, H., Lokstein, H., Dörmann, P., Grimm, B., and Benning, C. 1997. Changes in the composition of the photosynthetic apparatus in the galactolipid-deficient dgdl mutant of Arabidopsis thaliana. Plant Physiol. 115:1175-1184.

13. Iba, K. 2002. Acclimative response to temperature stress in higher plants: Approaches of gene engineering for temperature tolerance. Annu. Rev. Plant Biol. 53:225-245.

14. Jarvis, P., Dörmann, P., Peto, C. A., Lutes, J., Benning, C., and Chory, J. 2000. Galactolipid deficiency and abnormal chloroplast development in the Arabidopsis MGD synthase 1 mutant. Proc. Natl. Acad. Sci. USA 97:8175-8179.

15. Kim, Y. M., Bouras, N., Kav, N. N., and Strelkov, S. E. 2010. Inhibition of photosynthesis and modification of the wheat leaf proteome by Ptr ToxB: a host-specific toxin from the fungal pathogen Pyrenophora triticirepentis. Proteomics 10:2911-2926.

16. Lamari, L., and Bernier, C. C. 1989. Evaluation of wheat lines and cultivars to tan spot (Pyrenophora tritici-repentis) based on lesion type. Can. J. Plant Pathol. 11:49-56.

17. Lamari, L., and Strelkov, S. E. 2010. The wheat/Pyrenophora triticirepentis interaction: Progress towards an understanding of tan spot disease. Can. J. Plant Pathol. 32:4-10.

18. Loughman, R., and Deverall, B. J. 1986. Experiments with excised lesions from wheat leaves infected by Pyrenophora tritici-repentis. Australas. Plant Pathol. 18:90-93.

19. Maffei, M. E., Mithofer, A., and Boland, W. 2007. Before gene expression: Early events in plant-insect interaction. Trends Plant Sci. 12:310-316

20. Manning, V. A., and Ciuffetti, L. M. 2005. Localization of Ptr ToxA produced by Pyrenophora tritici-repentis reveals protein import into wheat mesophyll cells. Plant Cell 17:3203-3212.

21. McMullen, M., and Adhikari, T. 2009. Fungal leaf spot diseases of wheat: tan spot, Stagonospora nodorum blotch and Septoria tritici blotch. N. Dak. State Univ. Ext. Serv. PP-1249.

22. McMullen, M. P. 2010. Tan spot (yellow leaf spot). Pages 82-84 in: Compendium of Wheat Diseases and Pests. W. W. Bockus, R. L. Bowden, R. M. Hunger, W. L. Morrill, T. D. Murray, and R. W. Smiley, eds. American Phytopathological Society, St. Paul, MN

23. Moellering, E. R., Muthan, B., and Benning, C. 2010. Freezing tolerance in plants requires lipid remodeling at the outer chloroplast membrane. Science 330:226-228.

24. Murata N., Ishizaki-Nishizawa, O., Higashi, S., Hayashi, H., Tasaka, Y., and Nishida, I. 1992. Genetically engineered alteration in the chilling sensitivity of plants. Nature 356:313-326.

25. Raymond, P. J., Bockus, W. W., and Norman, B. L. 1985. Tan spot of winter wheat: Procedures to determine host response. Phytopathology 75:686-690.

26. Reifarth, F., Christen, G., Seeliger, A. G., Dörmann, P., Benning, C., and Renger, G. 1997. Modification of the water oxidizing complex in leaves of the dgdl mutant of Arabidopsis thaliana deficient in the galactolipid digalactosyldiacylglycerol. Biochemistry 36:11769-11776.

27. Shabeer, A., and Bockus, W. W. 1988. Tan spot effects on yield and yield components relative to growth stage in winter wheat. Plant Dis. 72:599602 .

28. Shah, J. 2005. Lipids, lipases, and lipid-modifying enzymes in plant disease resistance. Annu. Rev. Phytopathol. 43:229-260.

29. Singh, P. K., and Hughes, G. R. 2005. Genetic control of resistance to tan necrosis induced by Pyrenophora tritici-repentis, races 1 and 2, in spring and winter wheat genotypes. Phytopathology 95:172-177.

30. Strelkov, S. E., and Lamari, L. 2003. Host-parasite interaction in tan spot [Pyrenophora tritici-repentis] of wheat. Can. J. Plant Pathol. 25:339-349.

31. Strelkov, S. E., Lamari, L., and Balance, G. M. 1998. Induced chlorophyll degradation by a chlorosis toxin from Pyrenophora tritici-repentis. Can. J. Plant Pathol. 20:428-435.

32. Tomas, A., Feng, G. H., Reeck, G. R., Bockus, W. W., and Leach, J. E. 1990. Purification of a cultivar-specific toxin from Pyrenophora triticirepentis, causal agent of tan spot of wheat. Mol. Plant-Microbe Interact. 3:221-224

33. Tuori, R. P., Wolpert, T. J., and Ciuffetti, L. M. 1995. Purification and immunological characterization of toxic components from cultures of Pyrenophora tritici-repentis. Mol. Plant-Microbe Interact. 8:41-48. 
34. USDA-NASS. 2010. Wheat varieties. Online publication. USDA-NASS, Kansas Field Office, Department of Agriculture, Topeka, KS.

35. Voet, D., Voet, J. G., and Pratt, C. W. 2006.Biological membranes and lipids. Pages 234-281 in: Fundamentals of Biochemistry. Wiley, New York

36. Wang, X., Devaiah, S. P., Zhang, W., and Welti, R. 2006. Signaling functions of phosphatidic acid. Prog. Lipid Res. 45:250-278.

37. Welti, R., Li, W., Sang, Y., Biesiada, H., Zhou, H. E., Rajashekar, C. B., Williams, T. D., and Wang, X. 2002. Profiling membrane lipids in plant stress responses. Role of phospholipase D alpha in freezing-induced lipid changes in Arabidopsis. J. Biochem. 277:31994-32002.

38. Wolter, F. P., Schmidt, R., and Heinz, E. 1992. Chilling sensitivity of Arabidopsis thaliana with genetically engineered membrane lipids.
EMBO J. 11:4685-4692

39. Xiao, S., Gao, W., Chen, Q.-F., Chan, S.-W., Zheng, S.-X., Ma., J., Wang, M., Welti, R., and Chye, M.-L. 2010. Overexpression of Arabidopsis acylCoA binding protein ACBP3 promotes starvation-induced and agedependent leaf senescence. Plant Cell 22:1463-1482.

40. Yamauchi, Y., Furutera, A., Seki, K., Toyoda, Y., Tanaka, K., and Sugimoto, Y. 2008. Malondialdehyde generated from peroxidized linolenic acid causes protein modification in heat-stressed plants. Plant Physiol. Biochem. 46:786-793

41. Zhang, W., Qin, C., Zhao, J., and Wang, X. 2004. Phospholipase Da1derived phosphatidic acid interacts with $\mathrm{ABI} 1$ phosphatase $2 \mathrm{C}$ and regulates abscisic acid signaling. Proc. Natl. Acad. Sci. USA 101:95089513. 